\title{
When increasing population density can promote the evolution of metabolic cooperation
}

\author{
Richard J. Lindsay ${ }^{1}$ Bogna J. Pawlowska' • Ivana Gudelj
}

Received: 22 May 2017 / Revised: 13 September 2017 / Accepted: 30 October 2017 / Published online: 12 January 2018

(c) International Society for Microbial Ecology 2018

\begin{abstract}
Microbial cooperation drives ecological and epidemiological processes and is affected by the ecology and demography of populations. Population density influences the selection for cooperation, with spatial structure and the type of social dilemma, namely public-goods production or self-restraint, shaping the outcome. While existing theories predict that in spatially structured environments increasing population density can select either for or against cooperation, experimental studies with both public-goods production and self-restraint systems have only ever shown that increasing population density favours cheats. We suggest that the disparity between theory and empirical studies results from experimental procedures not capturing environmental conditions predicted by existing theories to influence the outcome. Our study resolves this issue and provides the first experimental evidence that high population density can favour cooperation in spatially structured environments for both self-restraint and public-goods production systems. Moreover, using a multi-trait mathematical model supported by laboratory experiments we extend this result to systems where the self-restraint and public-goods social dilemmas interact. We thus provide a systematic understanding of how the strength of interaction between the two social dilemmas and the degree of spatial structure within an environment affect selection for cooperation. These findings help to close the current gap between theory and experiments.
\end{abstract}

\section{Introduction}

Microorganisms engage in an impressive array of cooperative behaviours [1] that drive ecosystem and epidemiological processes including nutrient recycling [2], antibiotic resistance [3] and disease virulence [4-6]. These social interactions are shaped by the ecology and demography of populations with population density known to affect selection for cooperation [7-12].

Whether high-population density favours cooperation can depend on the spatial structure of the environment $[8$, $10]$ and the type of social dilemma faced [8-11]. In particular, two types of social dilemmas have been considered:

Richard J. Lindsay and Bogna J. Pawlowska contributed equally to this work.

Electronic supplementary material The online version of this article (https://doi.org/10.1038/s41396-017-0016-6) contains supplementary material, which is available to authorized users.

Ivana Gudelj

I.Gudelj@exeter.ac.uk

1 University of Exeter, Exeter, UK public-good production and self-restraint. Public-goods are extracellular factors used to perform a range of functions including nutrient acquisition, biofilm formation and quorum sensing [13]. They are costly to produce and benefit individuals in the locality. Therefore, public-goods are prone to exploitation by cheats who do not contribute to their production, but can still reap the rewards. Self-restraint cooperation arises from a metabolic trade-off between growth rate and efficiency, whereby fast growth is consequently less efficient than slow growth [14]. Efficient use of common resources conforms to the classical definition of a cooperative trait; it is beneficial to the group because more biomass is produced per unit of resource, but costly to individuals because they reproduce at a slower rate. This is prone to exploitation by cheats that use resources quickly but inefficiently for their own rapid multiplication, at the expense of the total population yield [10].

The relationship between population density and cooperation is not yet fully understood with theory and empirical studies in disagreement. Theory suggests that highpopulation density could favour either cooperators or cheats. For self-restraint cooperation it is argued that selection depends on the biological details of the system 
because density alters numerous factors including the resource availability per individual, the variation in resource concentrations as they are consumed, and how beneficial cooperation is [11]. For public-goods systems, it is postulated that high population density could favour cheats because in dense populations cheats are 'physically closer' to cooperators and can thus exploit them more effectively [11]. However it is also suggested that high cell density could favour cooperators if cells diffuse slower than the public-goods they produce [8], or the environment is sufficiently spatially structured $[15,16]$.

Until now empirical studies have only been able to demonstrate that in structured environments high population density favours cheats both in self-restraint [10] and publicgood $[7,9,11,12,17,18]$ cooperative systems. Moreover, contrary to the predictions in [8] experiments with motile [11] and non-motile [9] cell populations producing publicgoods both report the same outcome that high density favours cheats, even when cells diffuse slower than the public-goods.

Could the discrepancies between theoretical and empirical studies result from experimental procedures not capturing the extent of environmental variation that is predicted to affect the outcome ( $[8,11]$ To address this, we conducted a series of microbial laboratory experiments using a model cooperative system with Saccharomyces cerevisiae which exhibits both public-good [19] and selfrestraint cooperation [10]. We manipulated factors suggested by theoretical models to underpin the relationship between population density and cooperation, these are: the relative cost and benefits of cooperation $[8,11]$, the physical proximity of cells to each other [11] and the diffusion of public-goods and cells [8]. For the first time we provided experimental evidence supporting theoretical predictions that high population densities could favour cooperation in spatially structured environments in both self-restraint [11] and public-goods cooperative systems [8].

As cooperative traits frequently interact [5, 20-23] we next asked whether high population density can favour cooperation in systems where the self-restraint and publicgoods social dilemmas interact. To this end we developed the multi-trait mathematical model, manipulated the strength of interactions between the two social traits and showed that high population density can still favour cooperation. Moreover, our model predicted that decreasing the influence of self-restraint over public-goods production will increase the range of environmental structures where high population density favours cooperation. We provided a mechanistic explanation for this outcome, suggesting that the cost of inefficient metabolism arising in the presence of the self-restraint dilemma outweighs the benefit of publicgood cooperation in environments with low spatial structure where public-goods are more accessible to cheats. However, reducing the strength of the self-restraint dilemma will reverse the cost/benefit relationship in favour of cooperation. This was verified experimentally.

Our work provides the first empirical evidence that high population density can favour cooperation in spatially structured environments in both single and multi-trait cooperative systems and we provide an explanation as to why this result has so far been elusive.

\section{Materials and methods}

\section{The experimental system}

To secure nutrients microbes can cooperatively secrete enzymes, termed public goods, that break down complex sugars into simple sugars that are easier to digest $[24,25]$. Public-good cooperation in $S$. cerevisiae arises from the production of invertase to externally hydrolyse sucrose into glucose and fructose, the preferential carbon sources [24].

Once simple sugars are available in the environment, microbes are constrained by a metabolic trade-off between growth rate and efficiency [14], which is at the core of the self-restraint social dilemma. In S.cerevisiae, the rate at which resources are taken up alters whether they are catabolised by rapid, low yielding fermentation or slower, more efficient respiration [26, 27].

There is an inevitable interaction between public-good and self-restraint cooperation during invertase-mediated metabolism of sucrose by S. cerevisiae [21]. This occurs because when invertase secreting cells externally hydrolyse sucrose, they form local spikes in monosaccharide concentrations. Cells exposed to high resources concentrations metabolise them relatively less efficiently than when exposed to lower concentrations [27, 28], such as in the regions of cells that do not produce invertase. This forms the basis of self-restraint cooperation. Therefore, varying resource supply can control the strength of interaction between self-restraint and public-goods dilemmas. When resources are scarce, the rate-efficiency trade-off is weak $[21,28]$ and public-good production is expected to be the dominant constraint on growth. However, when resources are abundant, inefficient metabolism from high uptake rates will constrain growth [21].

\section{Mathematical model}

A mathematical model was developed to examine the relationship between population density and the interaction between self-restraint and public goods cooperation. The model was based on the established mathematical framework developed previously [21] with spatial interactions 


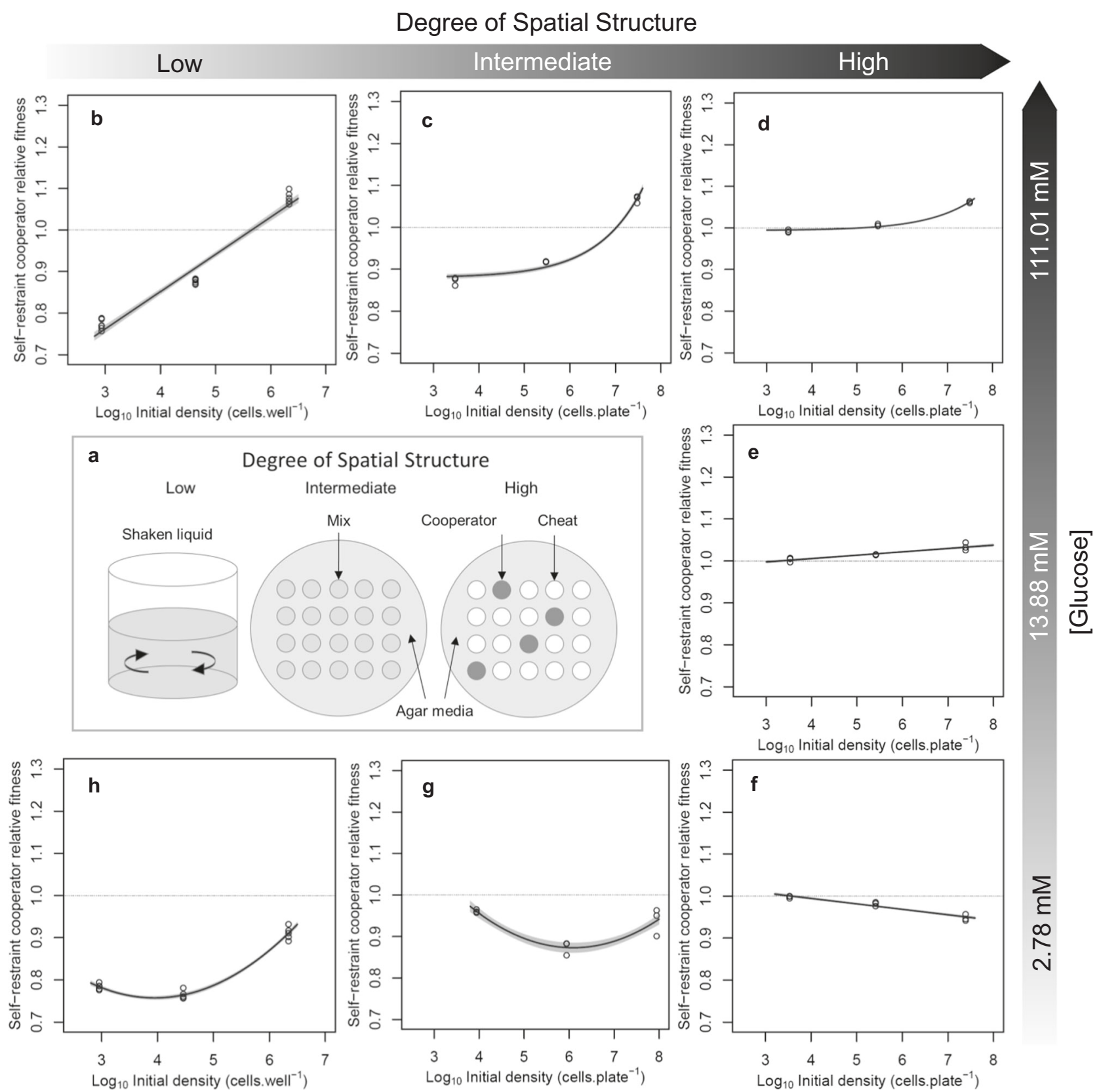

represented by a system of reaction-diffusion equations (For more details see Supplementary Text).

\section{Experimentally manipulating spatial structure of the environment}

We established the lowest level of structure in shaken liquid cultures (Fig. 1a). Note that while for self-restraint systems shaken liquid cultures represent spatially unstructured environments [10], for public-goods systems spatial structure is not completely absent. This is because invertase producers get preferential access to the public-good [19] and form small clumps when dividing [29]. An intermediate level of structure was established with initially mixed subpopulations on agar plates, and a high level was established with segregated subpopulations on agar plates (Fig. 1a).

\section{Strains}

Strains of $S$. cerevisiae were those used previously to test public-good cooperation [18, 19] (from J. Gore, MIT) (Fig. 2). The invertase producing strain (cooperator, BY4741, SUC2) constitutively expresses yEYFP by the TEF1 promoter. The non-invertase producing strain (JG210C, cheat, $\Delta$ suc2::kanMX4) expresses tdTomato by the $P G K 1$ promoter. 
Fig. 1 The relationship between self-restraint cooperator fitness and population density depends on the degree of spatial structuring in the environment and the extent of the rate-efficiency trade-off. a Schematic of degrees of spatial structure used for experiments. Low is shaken liquid, intermediate is patches of mixed strains, and high is made up of patches of either cooperators or cheats (for details see Supplementary Figure S1). In a low level of spatial structuring we found a positive relationship between density and cooperator fitness $(p$ $<1.06 \times 10^{-12}, \quad F_{(1,16)}=395.1, \quad$ Adj $R^{2}=0.9586, \quad \beta=0.08962 \pm$ 0.00451 (s.e.m.), $n=6, f=0.3$ ) (b). When competed on agar plates, the relationship was also positive for both intermediate $\mathbf{c}(\mathrm{p}<2.66 \times$ $10^{-7}, \quad \mathrm{~F}_{(1,7)}=365.7, \quad$ Adj $R^{2}=0.9785, \quad \beta=1.07 \pm 0.0560 \times 10^{-5}$ (s.e.m.), $n=3, f=0.3)$ and high structure $\mathbf{d}\left(p<8.56 \times 10^{-8}, F_{(1,7)}=\right.$ 508.2, Adj $R^{2}=0.9845, \beta=3.84 \pm 0.170 \times 10^{-5}$ (s.e.m.), $n=3, \mathrm{f}=$ 0.3 ), but the strength of this association reduced (from intermediate to high, interaction term: $\left.p<0.0002, \mathrm{~F}_{(1,14)}=25.11\right)$. With high structure, the relationship between density and cooperator fitness depended on the resource concentration. It was positive when glucose concentration was high $(111.01 \mathrm{mM})$, and remained positive with intermediate glucose $(13.88 \mathrm{mM})$ e $\left(p<0.0004, F_{(1,7)}=40.02\right.$, Adj $R^{2}=$ $0.8299, \quad \beta=0.00803 \pm 0.00127$ (s.e.m.), $n=3, f=0.3$ ), but the strength of this association was reduced (interaction term: $p<0.00276$, $\left.F_{1,14)}=13.141\right)$. However, this relationship became negative when glucose concentrations were further reduced $(2.78 \mathrm{mM}) \mathbf{f}(p<3.34 \times$ $10^{-5}, \quad F_{(1,7)}=87.34, \quad$ Adj $\quad R^{2}=0.9152, \quad \beta=-0.0130 \pm 0.00139$ (s.e.m.), $n=3, f=0.3)$. When resources were reduced $(2.78 \mathrm{mM})$ with an intermediate level of structure, we found regions of positive and negative density-dependence $\mathbf{g}$ (quadratic fit: $p<5.83 \times 10^{-3}, F_{(2,6)}=$ 13.68, Adj $\left.R^{2}=0.7601, n=3, f=0.2\right)$ with cooperator relative fitness lower at intermediate cell densities $\left(\sim 10^{6}\right.$ cells $)$ compared to low $\left(\sim 10^{4}\right.$, mean difference \pm s.e.m. $\left.=0.0871 \pm 0.0173, p<2.37 \times 10^{-3}\right)$ and high $\left(\sim 10^{8}\right.$, mean difference \pm s.e.m. $=0.0645 \pm 0.0173, \mathrm{p}<9.70 \times$ $10^{-3}$ ). With low structure, regions of positive and negative densitydependence were found in $2.78 \mathrm{mM}$ glucose $\mathbf{h}$ (quadratic fit: $p<$ $1.61 \times 10^{-10}, F_{(2,12)}=251.3$, Adj $\left.R^{2}=0.9728, n=5, f=0.3\right)$, cooperator relative fitness is lower at intermediate cell densities $\left(\sim 3 \times 10^{4}\right.$ cells) compared to low $\left(\sim 10^{3}\right.$, mean difference \pm s.e.m. $=0.0196 \pm$ $0.00709, p<0.0173)$ and high $\left(\sim 2 \times 10^{6}\right.$, mean difference \pm s.e.m. $=$ $\left.0.146 \pm 0.00709, p<9.62 \times 10^{-11}\right)$

Strains to test self-restraint cooperation were those used previously [10] (from P. Dahl, University of Gothenburg). CEN.PK2-1C has wild-type hexose transport capabilities and is a self-restraint cheat. Whereas TM6*, a mutant that has a single synthetic hexose transporter, is a self-restraint cooperator. The strains are distinguishable by fluorescence with CEN.PK2-1C constitutively expressing GFP and TM6* expressing mCherry, both by the TEF1 promoter, inserted into the URA3 locus. TM6* was also used as a public-good cooperator with reduced hexose uptake ability (Fig. 3d), but with a GFP marker.

To test the influence of interacting cooperative traits, in addition to competing BY4741 and JG210C (Fig. 4b, c), a non-invertase producing mutant of TM6* ( $\Delta$ suc2:: kanMX4) (Supplementary Figure S2) was generated in the mCherry-expressing background to compete with TM6* (GFP) (Fig. 4d-f).

Details of strains are summarised in Table 1. Strains used in each competition are also indicated in Figures.

\section{Competition experiments}

To experimentally test the influence of population density on the selection of cooperation in different environmental conditions, competition experiments were conducted in each environment with three different initial population densities. The nature of the relationship between cooperator fitness and density was assessed for each environment. The initial frequency of cooperators (f, given in figure legends) was equivalent for different spatial structures and resources concentrations tested, except when specifically testing the effect of changes in frequency on the relationship between density and cooperation (Supplementary Figure S5).

Competition experiments were conducted in defined media (DM: $6.9 \mathrm{~g} / 1$ Yeast Nitrogen Base w/o amino acids, $790 \mathrm{mg} / \mathrm{l}$ complete supplement mixture (Formedium, UK) with varying specified concentrations of glucose (for selfrestraint cooperation) or sucrose (for public-good and interacting cooperative traits) and $16 \mathrm{~g} / \mathrm{l}$ agar where applicable.

Strains were initially grown in $5 \mathrm{ml}$ YPD $(10 \mathrm{~g} / \mathrm{l}$ yeast extract, $20 \mathrm{~g} / \mathrm{l}$ peptone, $20 \mathrm{~g} / 1$ glucose) shaken overnight at $30{ }^{\circ} \mathrm{C}$. Cells were washed twice and resuspended in $5 \mathrm{ml}$ DM lacking sugar. Spatially structured competitions (intermediate and high) were performed on $9 \mathrm{~cm}$ diameter Petri dishes containing $25 \mathrm{ml}$ DM. Cells were inoculated onto agar plates in patches of $20 \mu \mathrm{l}$ in a $4 \times 5$ array (Fig. 1a), as described previously [21]. See Supplementary Figure S1 for details. Plates were sealed with parafilm to minimise evaporation and incubated at $30^{\circ} \mathrm{C}$ for $7 \mathrm{~d}$ to allow resources to diffuse and be consumed. Cells were collected by flooding the plates with $5 \mathrm{ml}$ PBS and colonies were gently scraped from the agar into suspension and appropriately diluted for flow cytometry to distinguish strain densities as detailed below. Each plate was considered a single replicate.

Low structure competitions were performed in DM, omitting agar, within a 48-well suspension culture microplate (Bio-One Greiner) $(640 \mu \mathrm{l}$ per well). Cultures were incubated at $30{ }^{\circ} \mathrm{C}$ in a FLUOstar Omega microplate reader (BMG Labtech) with shaking at 700 r.p.m. for periods to allow population growth to reach approximately stationary phase based on $\mathrm{OD}_{620 \mathrm{~nm}}$ readings. This was for $48 \mathrm{~h}$ for all competitions, except for those with low density and resources $(96 \mathrm{~h})$ and using TM6* $(72 \mathrm{~h})$ where growth rate was low owing to the Allee effect [30] and metabolic constraints [26].

Rate-efficiency trade-off tests were performed the same way for $72 \mathrm{~h}$, with an initial density of $2 \times 10^{5}$ cells well $^{-1}$. Population density was calibrated to OD for this system according to [30]. For our spectrophotometer, $O D_{b}=0.064$, $O D_{\max }=3.3$. For liquid cultures, each well was considered a replicate. Flow cytometry was used to establish the initial 

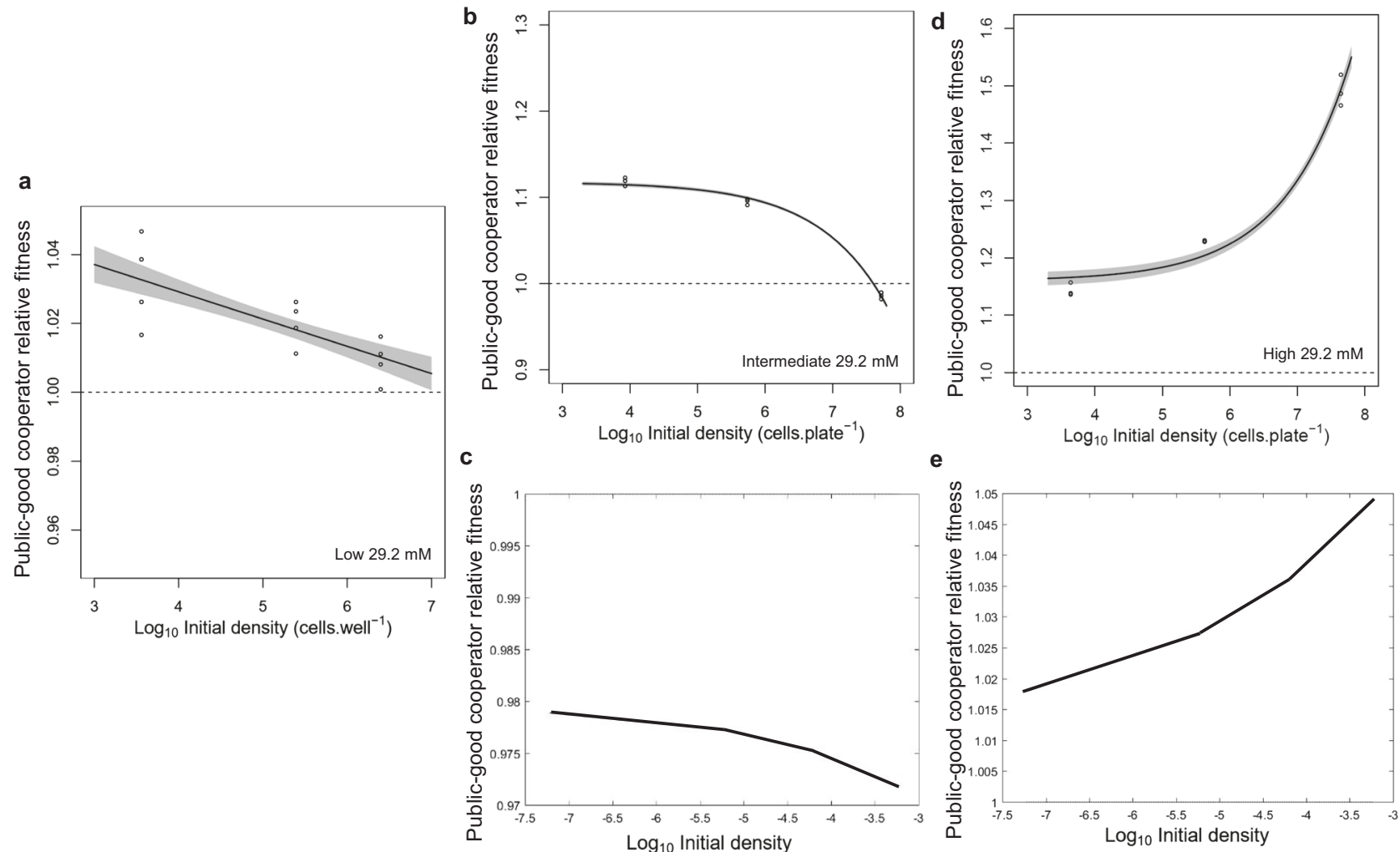

Fig. 2 The influence of population density and spatial structuring on the selection for public-good cooperation. The selection for publicgood cooperation (BY4741 v JG210C) is negatively density-dependent when the spatial structuring of the environment is low $\mathbf{a}(\mathrm{p}<0.00482$, $\mathrm{F}_{(1,10)}=12.99, \beta( \pm$ s.e.m. $)=-0.00793 \pm 0.00220$, Adj $R^{2}=0.5214$, $n=4, f=0.2)$ and intermediate $\mathbf{b}\left(p<6.98 \times 10^{-9}, F_{(1,7)}=1047, \beta(\right.$ \pm s.e.m. $)=-5.88 \pm 0.182 \times 10^{-5}$, Adj. $\left.R^{2}=0.992, n=3, f=0.2\right)$, as predicted by model simulations (Degree of Spatial Structuring (DSS) $=0$, see Supplementary Information for definition) c, and positively

and final densities of the strains. This was performed with a Guava easyCyte HT System using Guava InCyte software (Merck Millipore). Populations were diluted in PBS $(\mathrm{pH}$ 7.4) to a density of $10^{4}-5 \times 10^{5}$ cell ml $^{-1}$. Density was established by measuring events gated on FSC and SSC. Cooperator and cheat cells were gated based on fluorescence (Supplementary Figure S3). Relative fitness was calculated based on the ratio of Malthusian growth parameters [31], as done previously when assessing the densitydependent nature of cooperator fitness [9], with relative fitness $=1$ denoting equal fitness (see Supplementary text for details).

\section{Data analysis}

Statistical tests were performed using $\mathrm{R}$ version 3.1.1. We assess the nature and strength of the relationship between density $(x)$ and cooperator fitness (y) using linear models. Linear models were calculated and plotted density-dependent when structure is high $\mathbf{d}\left(\mathrm{p}<7.93 \times 10^{-7}, \mathrm{~F}_{(1,7)}=\right.$ 266.1, $\beta( \pm$ s.e.m. $)=1.60 \pm 0.0981 \times 10^{-4}$, Adj. $R^{2}=0.971, n=3, f$ $=0.2)$ as predicted by model simulations $(D S S=0.8)(\mathbf{e})$. Labels indicate level of structuring and [sucrose]. Experimental repeats generated equivalent qualitative trends, which were also found to be robust to differing initial frequencies of public-good cooperators (Supplementary Figure S5). For simulations, initial density is $\mathrm{g}$ biomass $1^{-1}, S_{0}$ $=50, f=0.4$

using the 'MASS' package (version 7.3-35) and plotted (black line) \pm s.e.m. (shaded regions). Monotonic models were used to capture changes in selection across the range of initial densities tested. Monotonic models were fitted in the form $y=x$ or $y=e^{x}$ with the better model fit shown based on AIC values with coefficient estimates $(\beta)$ reported. Though not optimal fits, these were used for simplicity to readily distinguish switches in the selection for cooperation. The initial density was $\log _{10}$ transformed for the assumptions of the parametric statistical test. Comparisons of the strength of association between density and fitness between levels of structuring and resource concentrations (Fig. 1) were performed with a GLM with density and structure or resource concentration as explanatory variables with an interaction term between the two. Non-monotonic relationships were examined with quadratic terms, and assessed for best fit based on AIC values, with linear models using density as factor to assess relative fitness differences. 

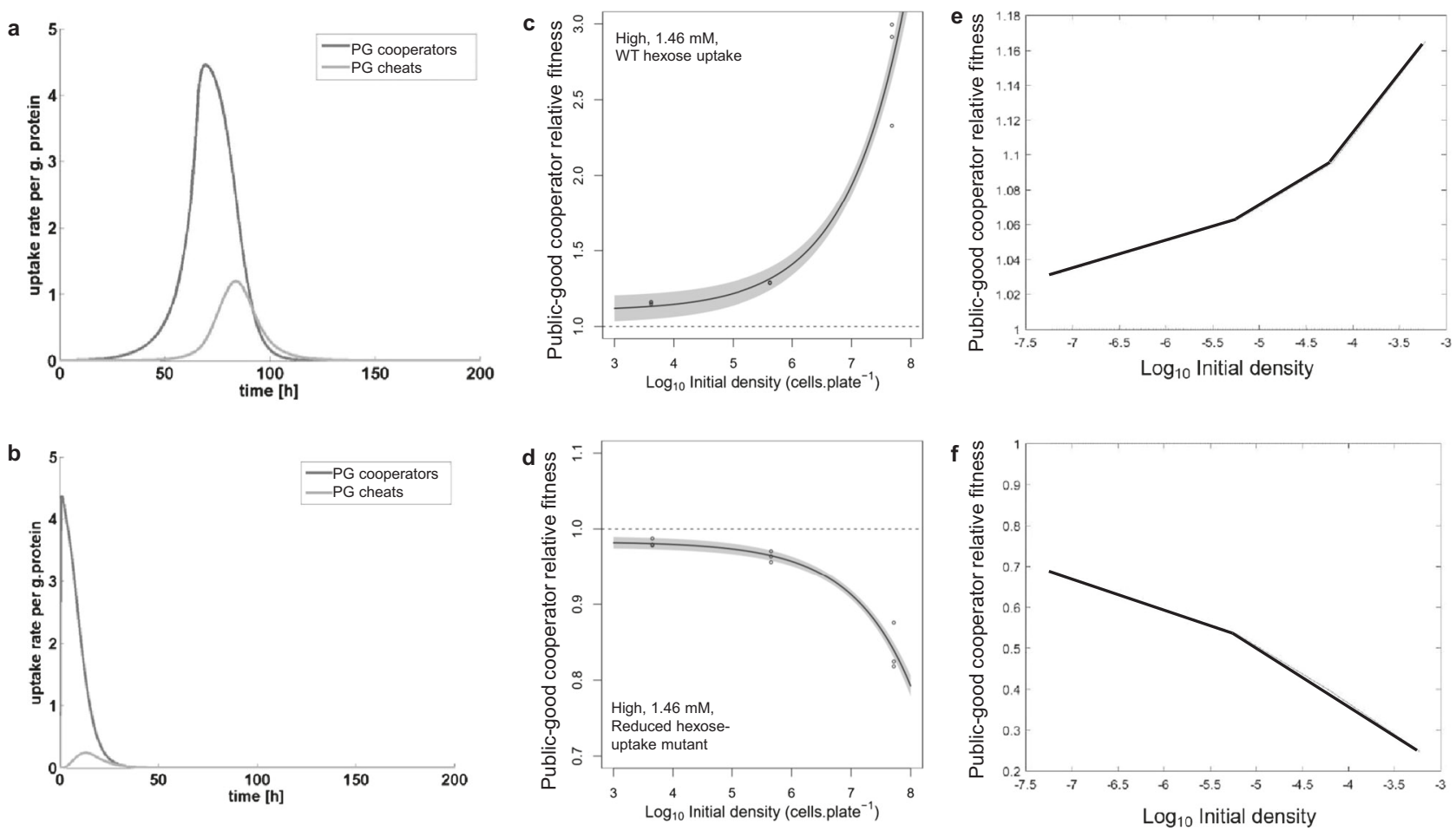

Fig. 3 Positive density-dependence of public-good cooperator fitness in structured environments results from a higher capacity to capture public-goods. Average hexose uptake rates (over all spatial locations) of public-good (PG) cooperators and cheats in a spatially structured environment at low population density $\left(N_{0}=6 \times 10^{-6} \mathrm{~g}\right.$ biomass $\left.1^{-1}\right)$ a and high population density $\left(N_{0}=6 \times 10^{-2} \mathrm{~g}\right.$ biomass $\left.1^{-1}\right) \mathbf{b}$, as predicted by model simulations ( $D S S=0.7, n=5, p=1, S_{0}=5.85$ ). The amount of hexose captured by PG cheats is higher in low-density than high-density populations. The selection for cooperation in $1.46 \mathrm{mM}$ sucrose is positively-density-dependent in highly structured environments with wild-type hexose capture ability (BY4741) $(p<1.12 \times 10$ ${ }^{-5}, \quad F_{(1,7)}=121.6, \quad \beta( \pm$ s.e.m. $)=7.57 \pm 0.686 \times 10^{-4}$, Adj. $R^{2}=$ $0.9378, n=3, f=0.3)$ c, but becomes negatively density-dependent when the capacity for public-good producers to capture the generated

\section{Results}

\section{Self-restraint cooperation}

Previous experimental studies found that density-dependent selection for self-restraint cooperation is positive in spatially unstructured yet negative in structured environments [10]. While our experiments agree for shaken liquid environments (Fig. 1b), in structured environments we found the opposite (Fig. 1c-d), namely that high population density favours cooperation. Why is our result different to that in ref. [10]. The difference comes from the way spatial structure is represented experimentally. In ref. [10], unshaken liquid cultures were used to represent a spatially structured environment while our experiments were conducted on agar plates (Fig. 1a).

We found that the extent to which increasing density selects for cooperation reduces as spatial structuring hexoses is reduced $\left(\right.$ TM6 $\left.^{*}\right)\left(p<1.29 \times 10^{-5}, F_{(1,7)}=116.6, \quad \beta\right.$ $( \pm$ s.e.m. $)=-6.40 \pm 0.593 \times 10^{-5}$, Adj. $\left.R^{2}=0.9353, n=3, f=0.3\right)$ (d). Labels indicate level of structuring, [sucrose], and the hexose uptake attributes of the public-good cooperator. Experimental repeat can be seen in Supplementary Figure S6. Based on growth rate data from this study, with calculations and invertase activity data from [19], we estimate that the glucose capture efficiency of TM6* is $69.6 \pm$ $0.939 \%$ of wild-type capabilities in $1.46 \mathrm{mM}$ sucrose (TM6* $<$ wildtype: $p<0.001, t=32.3, n=3$, Welch's two-tailed $t$-test). Our model predicts the same outcome for PG cooperator relative fitness in structured environments $\left(S_{0}=1.46, D S S=0.7, f=0.2\right.$, initial density is $\mathrm{g}$ biomass $1^{-1}$ ), with positive-density-dependence with wild-type hexose uptake $\mathbf{e}$ and negative density-dependence when hexose uptake is reduced $(V h / 10) \mathbf{f}$

increases (Fig. 1c, d). This result arises, as suggested [11], because spatial structuring alters the relative magnitude of benefits gained through cooperation. In our system, resources become heterogeneously distributed when the population is spatially structured because selfrestraint cooperators and cheats have disparate resource consumption rates [26]. These resource gradients alter the pay-offs of self-restraint cooperation through the rate-efficiency trade-off and/or the antagonistic metabolic by-products generated by self-restraint cheats [10, 28]. To test this, we repeated competitions when spatial structuring was high, but we altered the cost-to-benefit ratio of self-restraint cooperation by reducing the resource concentration (Supplementary Figure S4). When glucose concentration lowered, from 111.01 to $13.88 \mathrm{mM}$, positive density-dependence of self-restraint cooperator fitness remained, however the strength of this relationship was lessened (Fig. 1e). When reduced further (to $2.78 \mathrm{mM}$ ), 
Table 1 Strains used in this study with invertase (SUC2), hexose uptake and fluorescence marker attributes

\begin{tabular}{lllll}
\hline Strain & $\begin{array}{l}\text { Cooperation } \\
\text { reference }\end{array}$ & Invertase & Hexose uptake & Fluorescence \\
\hline BY4741 & {$[18]$} & $S U C 2$ & wild-type \\
JG210C & {$[19]$} & $\begin{array}{l}\Delta s u c 2:: \\
\text { kanMX }\end{array}$ & wild-type & yeYFP \\
& & $S U C 2$ & $\begin{array}{l}\text { wild-type }(\geq 20 \text { hexose } \\
\text { transporters })\end{array}$ & GFP \\
CEN.PK2-1C & {$[10]$} & sUC2 & single hexose transporter & mCherry \\
TM6* (mCherry) & {$[10]$} & $S U C 2$ & single hexose transporter & GFP \\
TM6* (GFP) & {$[10]$} & $\Delta s u c 2::$ & single hexose transporter & mCherry \\
TM6* $\Delta$ suc2 & This study & kanMX4 & & \\
\hline
\end{tabular}
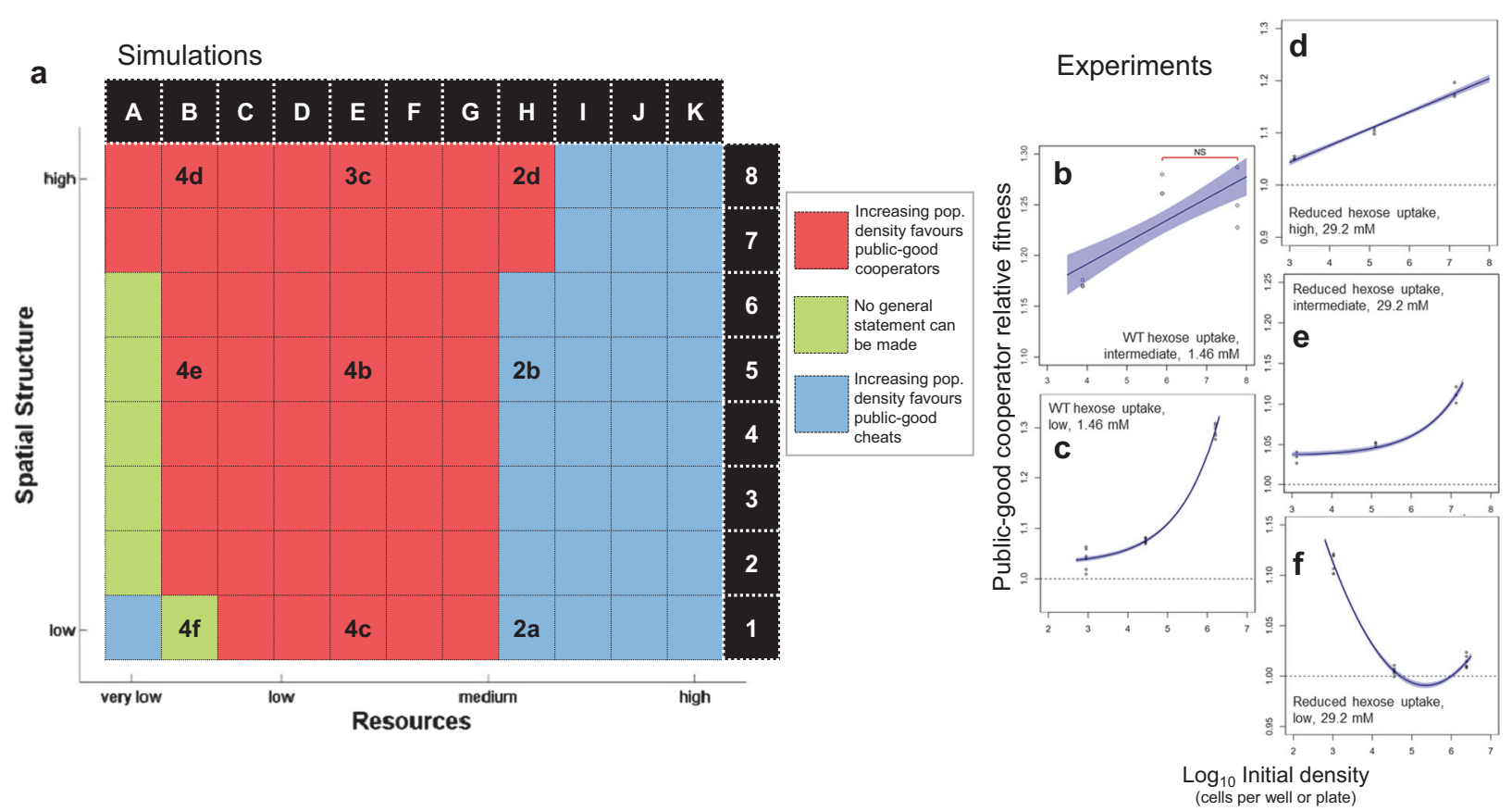

Fig. 4 The effect of population density on public-good cooperation as a function of spatial structure and strength of rate-efficiency trade-off: simulations and empirical results. a Competitions were simulated between public-good cooperators and cheats in 88 environmental conditions ( 8 degrees of spatial structure in 11 resource concentrations) at three initial population densities. The outcomes of the competitions in relation to increasing population density are illustrated in the matrix (yellow panels: increasing density may favour co-operators or cheats depending on the details of the system). See Supplementary Information for details on how the outcome of numerical simulations were assessed. Labels within panels indicate the figure in this article that represents experimental support that replicates predictions from the mathematical model. Predictions of the simulation were tested experimentally (b-f; labels within plots indicate hexose uptake ability of both competitors, degree of structure and [sucrose]). b When the impact of the rate-efficiency trade-off is reduced by lowering resources (from 29.2 to $1.46 \mathrm{mM}$ sucrose) we find public-good cooperator fitness becomes positively density-dependent with intermediate spatial structure $\left(p<0.0173, \mathrm{~F}_{(1,7)}=9.613, \beta( \pm\right.$ s.e.m. $)=0.0215 \pm 0.00694$, Adj $\left.R^{2}=0.5185, n=3, f=0.2\right)$. Test for non-monotonicity is notsignificant (NS, $p>0.434$ ) (c.f. Figure 2b). c With low structure at

$1.46 \mathrm{mM}$, public-good cooperator fitness becomes positively densitydependent $\left(p<2.42 \times 10^{-16}, F_{(1,16)}=1154, \beta( \pm\right.$ s.e.m. $)=5.38 \pm$ $0.158 \times 10^{-4}$, Adj $R^{2}=0.9855, n=6, f=0.2$ ) (c.f. Figure 2a). d In competitions between exclusively respiring public-good cooperators (TM6*) and cheats (TM6* $\Delta$ suc2) with high structure, public-good cooperator fitness was positively density-dependent $\left(p<1.50 \times 10^{-6}\right.$, $F_{(1,7)}=221$, Adj- $\left.R^{2}=0.9649, \beta=0.0321 \pm 0.00216, n=3, f=0.3\right)$ like wild-type hexose transporting strains (BY4741 v JG210C) (Fig. 2d). e However, unlike wild-type hexose transporting strains with intermediate structure (Fig. 2b) this relationship remained positive $(p<$ $4.25 \times 10^{-6}, F_{(1,7)}=162.4, \quad$ Adj- $R^{2}=0.9528, \beta=6.07 \pm 0.476 \times 10$ $\left.{ }^{-5}, n=0.3, f=0.3\right)$. These results were predicted by our model when hexose uptake rate is diminished (Supplementary Figure S7). f In low structure, we found that density has a non-monotonic relationship with public-good cooperator fitness (quadratic: $p<5.00 \times 10^{-14}, F_{(2,15)}=$ 437.7, Adj- $\left.R^{2}=0.9809, n=6, f=0.3\right)$. Public-good cooperator relative fitness was lower at intermediate densities $\left(\sim 5 \times 10^{4}\right)$ compared with low $\left(\sim 10^{3}\right.$ cells, mean difference \pm s.e.m. $=0.107 \pm 0.00399, p<$ $\left.4.64 \times 10^{-14}\right)$ and high density $\left(\sim 2.5 \times 10^{6}\right.$, mean difference \pm s.e.m. $=0.00923 \pm 0.00399, p<0.0356$, linear model with density as factor) 
self-restraint cooperator fitness was negatively densitydependent (Fig. 1f).

Therefore, as suggested by theory [11], we found that the relationship between self-restraint cooperator fitness and population density depended on biological details of the system, such as the relative pay-offs of self-restraint cooperation. This can be altered through spatial structuring, which alters the available resource concentrations and hence the incentives to cheat. To demonstrate this, we conducted competitions with low and intermediate levels of spatial structure and low resources $(2.78 \mathrm{mM})$. Unlike with high resource levels $(111 \mathrm{mM})$, we found regions of both positive and negative density-dependence of self-restraint cooperator fitness (Fig. 1g-h). This illustrates how the biological parameters dictate the way in which density influences the selection for self-restraint cooperation [11] and how resource concentration and spatial structure interact to dictate cooperator success.

\section{Public-good cooperation}

In agreement with previous experimental studies with this $S$. cerevisiae system, we found that for intermediate resource concentrations (29.2 mM sucrose) public-good cooperator fitness was negatively density-dependent when the level of spatial structuring was low (Fig. 2a) [7, 12] and intermediate (Fig. 2b) [9], a relationship that is predicted by our mathematical model (Fig. 2c, Supplementary Information). These findings also agree with bacterial public-goods systems $[11,17]$.

However, theory suggests that the relationship between density and public-good cooperator fitness depends on the degree of diffusion of public-goods and cells [8]. To examine this, we introduced a higher degree of spatial structuring (Fig. 1a, high structure), and hence altered the distances between strains over which public-goods diffuse. In that case, we found public-good cooperator fitness was positively density-dependent (Fig. 2d), in agreement with previous theory [8] and predictions made by our mathematical model (Fig. 2e).

Why can high population densities favour public-good cooperation? We reason that the higher the density of publicgood cooperators, the quicker they will consume the resources made available from public-good production. In our highly-structured environment the available hexose is sufficiently scarce and heterogeneously distributed. Therefore, at higher densities cooperators will consume a larger proportion of the resources before they diffuse away to become accessible to cheats, as suggested previously $[8,29]$. Our model illustrated this effect where in a highly-structured environment with sufficiently low resources, the overall amount of hexose captured by public-good cheats was higher at low density (Fig. 3a) than at high density (Fig. 3b).
To experimentally test this prediction we repeated competitions between public-good cooperators and cheats in the same highly spatially structured environment. However, this time we replaced the public-good cooperator (Wt S. cerevisiae possesses at least 20 hexose transporter genes [32]) with a public-good cooperator, TM6*, which has just a single hexose transporter and therefore its maximal hexose uptake rate is only about $10 \%$ of the Wt [26]. This meant that TM6* was less able to take advantage of the highdensity conditions to capture the liberated hexose from sucrose before it diffuses away. Competitions with the TM6* public-good cooperator and Wt public-good cheat (JG210C) were performed on $1.46 \mathrm{mM}$ sucrose to reduce the influence of the rate-efficiency trade-off [26] because at sufficiently low sucrose concentrations the rate-efficiency trade-off is diminished [28] (Supplementary Figure S6a-b). Unlike with the wild-type hexose transporter strain (Fig. 3c), the fitness of the TM6* public-good cooperator was negatively density-dependent (Fig. 3d). This outcome was also captured by our model when we reduced the maximal hexose uptake rate of the public-good cooperator to $10 \%$ of the wildtype (Fig. 3e, f). This switch from positive to negative density-dependence experimentally verifies that in structured environments as population density increases, public-goods cooperators have an increased capacity to capture hexose liberated from sucrose.

\section{Interacting effects between density, spatial structure, and the rate-efficiency trade-off on the evolution of public-good cooperation}

How is the relationship between population density and public-good cooperation influenced by a second social trait, namely self-restraint, given that these traits can interact [21]. To explore this we conducted numerical simulations of our multi-trait model (Supplementary Information) in environments with varying degree of spatial structure and resource concentration (Fig. 4a). The latter alters the strength of self-restraint dilemma [21] whereby low resource enviroments reduce the strength of the rateefficiency trade-off, which underpins self-restraint cooperation.

Our model predicted that whether density favours publicgood cooperation depends both on the spatial structure and resource concentration of that environment (Fig. 4a). We predicted that reducing the resource concentration could increase the range of spatial structures where increasing population density favours public-good cooperation (Fig. 4a columns $\mathrm{C}-\mathrm{G}$ vs. $\mathrm{H}-\mathrm{K})$. At higher resource concentrations, public-good cooperators incur a cost of inefficient metabolism because of local spikes in hexose concentration that are formed as they hydrolyse sucrose [21]. This cost outweighs the relative benefit of public-good cooperation when 
spatial structure is lower (Fig. 4a, H1:K6), as in that scenario public-goods are more accessible to cheats. However, reducing the strength of the self-restraint dilemma by reducing resources, will diminish the cost of inefficient metabolism incurred by public-good cooperators because the hexose concentration spikes formed will be less pronounced [21]. Therefore, in these environments increasing density will favour public-good cooperators even when spatial structure is low (Fig. 4a, C1:G6) because of an enhanced ability to capture resources before they diffuse away (Fig. 3a, b).

We tested this prediction by competing public-good cooperators and cheats with wild-type hexose uptake in environments with intermediate and low spatial structuring, where increasing density had earlier been found to favour public-good cheats (Fig. 2a, b), however this time with reduced resources. As predicted by our model (Fig. 4a columns $C-G$ ), reducing resources resulted in the fitness of public-good cooperators becoming positively densitydependent in both intermediate and low levels of spatial structure (Fig. 4b, c).

To verify that this direction of selection switch is caused by interactions between public-goods and self-restraint, instead of reducing the resource concentration we performed competitions between purely respiring, and hence metabolically efficient (Supplementary Figure S6a, b), invertase producers (TM6*) and non-producers (TM6* $\Delta s u c 2)$. Again in sufficiently structured environments, public-good cooperator fitness was positively densitydependent (Fig. 4d). However unlike with the wild-type respiro-fermenting strains (Fig. 2b), with intermediate levels of structure public-good cooperator fitness was positively density-dependent (Fig. 4d), as predicted by our model (Supplementary Figure S7). With low structure, a nonmonotonic relationship between density and public-good cooperator fitness was found (Fig. 4f). This result was predicted by our model when the rate-efficiency trade-off is absent at low resource concentrations where no general statement could be made regarding density and selection for cooperation (Fig. 4a).

\section{Discussion}

In this article we provided the first experimental evidence that high population density can favour microbial cooperation in spatially structured environments in both public good production and self-restraint cooperative systems, as well as when the two social dilemmas interact.

Past theories have predicted that in spatially structured environments increasing population density can either select for or against self-restraint cooperation depending on the details of the system, such as how beneficial cooperation is
[11]. Yet empirical studies show that high population density favours self-restraint cheats [10]. Similarly, for publicgoods systems theory predicts that high population density favours cheats when they are "physically closer" to cooperators [11] and favours cooperators if microbial cells diffuse slower than the public-goods [8] or the environment is sufficiently spatially structured $[15,16]$. However, empirical studies with public-good cooperative systems consistently show that high population density favours cheats $[7,9,11,12,17,33]$.

We postulated that the descrepancy between theory and experiments could be due to experimental procedures not capturing the extent of environmental variation predicted to affect the outcome $[8,11]$. Indeed, our theoretical model also showed that whether high population density promotes cooperation depends intricately on the degree of spatial structuring as well as resource concentration in the environment (Fig. 4a). However, while some empirical studies systematically vary the degree of spatial structure [17, 34], more frequently spatial structure is manipulated through an initial distribution of organisms within an environment. In such cases many "spatially heterogeneous" initial distributions all fall into the same category of "structured" environments. For instance, microbial communities can be fragmented into subpopulations that are linked only through migration. Such metapopulation structure can be imposed experimentally by embedding populations into microtitre plates and the initial spatial distribution and migration is controlled [7, 35]. Another way of manipulating spatial structure is to inoculate agar media with numerous droplets containing microbes, forming patches of interacting subpopulations $[5,11,21]$. In this scenario, the initial spatial distribution is controlled, but the subsequent interactions between subpopulations are not. Finally, homogeneous cultures can be inoculated: (a) onto agar media [4, 9, 11, 36, 37]; (b) into unshaken liquid cultures $[10,29,38]$ or (c) into shaken liquid cultures [12, 18], for certain public-goods systems where producers get preferential access to the public-good [19]. In all three cases $(\mathrm{a}-\mathrm{c})$ spatial structure self-emerges and both the initial spatial cell distribution and the subsequent interactions between the emergent subpopulations are not controlled. However, high population densities can impede the selfaggregation of cells [37].

We conducted laboratory experiments using a model cooperative system with $S$. cerevisiae which exhibits both public-good [19] and self-restraint cooperation [10]. We considered three levels of spatial structure and demonstrated that the relationship between population density and cooperation depends on the degree of spatial structure as well resource concentration within an environment (Figs. 1,4,2). Therefore, we argue that previous experimental studies with self-restraint [10] and public-goods [9, 11] cooperative 
systems found that high population density favours cheats because they were conducted in environments with either relatively low spatial structure or high resource concentration.

Our data supports the mechanistic explanations put forward by theoretical models as to why high population densities can favour cooperation. For self-restraint cooperation, the magnitude of benefits gained through cooperation influences the extent to which increasing density selects for cooperation [11]. In our system, resources become heterogeneously distributed when the population is spatially structured because self-restraint cooperators and cheats have disparate resource consumption rates [26]. These resource gradients mean that the pay-offs of self-restraint cooperation are altered through the rate-efficiency trade-off and/or the antagonistic metabolic by-products generated by selfrestraint cheats $[10,28]$.

Similarly, we demonstrated that with increasing population density public-good cooperation can either be selected for or against depending on whether the environment is sufficiently structured and how this structure influences the amount of public-good benefit gained. Intuitively, increasing population density increases the incentives to cheat because it increases the proximity to, and absolute number of, public-good cooperators that can be exploited [11]. On the other hand, increasing density also increases the amount of the public-goods that are generated, which can be captured by cooperators before they diffuse to become available to cheats [8]. The scale of population structuring will alter the relative extent of these opposing forces, which in turn will shape whether density selects for or against publicgood cooperation. We experimentally verified this mechanism by limiting the ability of public-good producers to obtain the generated benefits of public-good cooperation. This predictably switched the direction of selection from increasing density favouring public-good cooperators (Fig. 3c) to favouring cheats (Fig. 3d).

We also showed that high population density can favour cooperation even when the self-restraint and public-goods social dilemmas interact (Fig. 2d). This is important as cooperative traits frequently interact [5, 20-23]. We developed a multi-trait mathematical model and predicted that decreasing the influence of self-restraint over publicgoods production will increase the range of environmental structures where high population density favours cooperation (Fig. 4a columns $\mathrm{C}-\mathrm{G}$ vs. $\mathrm{H}-\mathrm{K}$ ). This was verified experimentally. For example, when resources were sufficiently low, high population density favoured cooperators in environments with low (Fig. 4c), intermediate (Fig. 4b) and high (Fig. 3c) spatial structure. However, for sufficiently high resource concentrations, high population density favoured cooperators only when spatial structure was high (Fig. 2d).
We reason that at higher resource concentrations, publicgoods cooperators incur a cost of inefficient metabolism. Therefore in environments with sufficiently low spatial structure where public-goods are more accessible to cheats, the cost of inefficient metabolism outweighs any personal benefit of public-goods production. Reducing the resource concentration and therefore reducing the strength of the self-restraint dilemma diminishes the cost of inefficient metabolism incurred by public-goods cooperators, tipping the balance in favour of cooperators even in environments with low spatial structure.

For certain environmental conditions our model predicts that increasing population density may favour public-good cooperators or cheats depending on the details of the system (Fig. 4a, yellow panels). This is in line with empirical observations of a non-monotone relationship between population density and cooperator relative fitness (Fig. 4f).

Our results are also of relevance to the study of cooperation in populations undergoing an increase in the geographical area they occupy, known as range expansion. Recent studies have found that the advantage public-good cooperators can gain at low density may enrich cooperation at the front of expanding populations [7]. Our results suggest that this may not be the case if the environment is sufficiently structured or has low resources. Microbes inherently exist in structured communities, whether as clonal patches such as colonies or hyphal networks [29, 39], or they inhabit spatially structured substrates [40, 41], which may frequently be nutrient depleted [42]. In these environments, rather than being promoted at the low-density expanding front of a population, cooperation may be more resistant against an invasion of cheats in the established bulk population where density is high as the community proliferates. Conversely, this means that if public-good cheats arise through mutation they may be more likely to gain a competitive advantage in the low-density advancing front of a population during range expansion. This "allele surfing" phenomenon may facilitate the maintenance of metabolic diversity within a population [43], even for potentially deleterious mutations [44], until environmental conditions become more preferential for public-good cheats. This diversity could enable adaptability to environmental change, improve the outcome of ecosystem processes [2], and maximise population fitness $[5,21]$.

Acknowledgements We would like to thank Robert Beardmore for comments and helpful discussions.

Funding RJL and IG: European Research Council No. 647292 MathModExp. BJP: Engineering and Physical Sciences Research Council Doctoral training grant studentship. 


\section{Compliance with ethical standards}

Conflict of interest The authors declare that they have no conflict of interest.

\section{References}

1 Crespi BJ. The evolution of social behavior in microorganisms. Trends Ecol Evol. 2001;16:178-83.

2 Kaiser C, Franklin O, Richter A, Dieckmann U. Social dynamics within decomposer communities lead to nitrogen retention and organic matter build-up in soils. Nat Commun. 2015;6:8960.

3 Lee HH, Molla MN, Cantor CR, Collins JJ. Bacterial charity work leads to population-wide resistance. Nature. 2010;467:82-85.

4 Griffin AS, West SA, Buckling A. Cooperation and competition in pathogenic bacteria. Nature. 2004;430:1024-7.

5 Lindsay RJ, Kershaw MJ, Pawlowska BJ, Talbot NJ, Gudelj I. Harbouring public good mutants within a pathogen population can increase both fitness and virulence. Elife. 2016; 5: e18678.

6 Crespi B, Foster K, Ubeda F. First principles of Hamiltonian medicine. Philos Trans R Soc Lond B Biol Sci. 2014;369:20130366.

7 Datta MS, Korolev KS, Cvijovic I, Dudley C, Gore J. Range expansion promotes cooperation in an experimental microbial metapopulation. Proc Natl Acad Sci USA. 2013;110:7354-9.

8 Dobay A, Bagheri HC, Messina A, Kümmerli R, Rankin DJ. Interaction effects of cell diffusion, cell density and public goods properties on the evolution of cooperation in digital microbes. $\mathbf{J}$ Evol Biol. 2014;27:1869-77.

9 Greig D, Travisano M. The Prisoner's Dilemma and polymorphism in yeast SUC genes. Proc Biol Sci. 2004;271:S25-26.

10 MacLean RC, Gudelj I. Resource competition and social conflict in experimental populations of yeast. Nature. 2006;441:498-501.

11 Ross-Gillespie A, Gardner A, Buckling A, West SA, Griffin AS. Density dependence and cooperation: theory and a test with bacteria. Evolution. 2009;63:2315-25.

12 Chen A, Sanchez A, Dai L, Gore J. Dynamics of a producerfreeloader ecosystem on the brink of collapse. Nat Commun. 2014;5:3713.

13 West SA, Diggle SP, Buckling A, Gardner A, Griffin AS. The social lives of microbes. Annu Rev Ecol Evol Syst. 2007;38:53-77.

14 Pfeiffer T, Schuster S, Bonhoeffer S. Cooperation and competition in the evolution of ATP-producing pathways. Science. 2001;292:504.

15 Lion S. Evolution of reproductive effort in viscous populations: the importance of population dynamics. J Evol Biol. 2010;23:866-74.

16 Lion S, Gandon S. Habitat saturation and the spatial evolutionary ecology of altruism. J Evol Biol. 2009;22:1487-502.

17 Kümmerli R, Griffin AS, West SA, Buckling A, Harrison F. Viscous medium promotes cooperation in the pathogenic bacterium Pseudomonas aeruginosa. Proc Biol Sci. 2009;276:3531-8.

18 Celiker H, Gore J. Competition between species can stabilize public-goods cooperation within a species. Mol Syst Biol. 2012;8:621.

19 Gore J, Youk H, van Oudenaarden A. Snowdrift game dynamics and facultative cheating in yeast. Nature. 2009;459:253-6.

20 Harrison F, Buckling A. Siderophore production and biofilm formation as linked social traits. ISME J. 2009;3:632-4.

21 MacLean RC, Fuentes-Hernandez A, Greig D, Hurst LD, Gudelj I. A mixture of "cheats" and "co-operators" can enable maximal group benefit. PLoS Biol. 2010; 8: e1000486.

22 Brown SP, Taylor PD. Joint evolution of multiple social traits: a kin selection analysis. Proc R Soc B. 2009;277;1680:415-422.
23 Ross-Gillespie A, Dumas Z, Kümmerli R. Evolutionary dynamics of interlinked public goods traits: an experimental study of siderophore production in Pseudomonas aeruginosa. J Evol Biol. 2015;28:29-39.

24 Schweizer M, Dickinson JR. The Metabolism and Molecular Physiology of Saccharomyces cerevisiae. CRC Press; 2004.

25 Talbot NJ. Living the sweet life: how does a plant pathogenic fungus acquire sugar from plants? PLoS Biol. 2010;8:e1000308.

26 Otterstedt K, Larsson C, Bill RM, Ståhlberg A, Boles E, Hohmann $\mathrm{S}$, et al. Switching the mode of metabolism in the yeast Saccharomyces cerevisiae. EMBO Rep. 2004;5:532-7.

27 Postma E, Verduyn C, Scheffers WA, Van Dijken JP. Enzymic analysis of the crabtree effect in glucose-limited chemostat cultures of Saccharomyces cerevisiae. Appl Environ Microbiol. 1989;55:468-77.

28 Weusthuis RA, Pronk JT, van den Broek PJ, van Dijken JP. Chemostat cultivation as a tool for studies on sugar transport in yeasts. Microbiol Rev. 1994;58:616-30.

29 Koschwanez JH, Foster KR, Murray AW. Sucrose utilization in budding yeast as a model for the origin of undifferentiated multicellularity. PLoS Biol. 2011;9:e1001122.

30 Dai L, Vorselen D, Korolev KS, Gore J. Generic indicators for loss of resilience before a tipping point leading to population collapse. Science. 2012;336:1175-7.

31 Lenski RE, Rose MR, Simpson SC, Tadler SC. Long-term experimental evolution in Escherichia coli. I. Adaptation and divergence during 2,000 generations. Am Nat. 1991;138:1315-41.

32 Wieczorke R, Krampe S, Weierstall T, Freidel K, Hollenberg CP, Boles E. Concurrent knock-out of at least 20 transporter genes is required to block uptake of hexoses in Saccharomyces cerevisiae. FEBS Lett. 1999;464:123-8.

33 Sanchez A, Gore J. Feedback between population and evolutionary dynamics determines the fate of social microbial populations. PLoS Biol. 2013;11:e1001547.

34 Boots M, Mealor M. Local interactions select for lower pathogen infectivity. Science. 2007;315:1284-6.

35 Dai L, Korolev KS, Gore J. Slower recovery in space before collapse of connected populations. Nature. 2013;496:355-8.

36 Le Gac M, Doebeli M. Environmental viscosity does not affect the evolution of cooperation during experimental evolution of colicigenic bacteria. Evolution. 2010;64:522-33.

37 van Gestel J, Weissing FJ, Kuipers OP, Kovács AT. Density of founder cells affects spatial pattern formation and cooperation in Bacillus subtilis biofilms. ISME J. 2014;8:2069-79.

38 Rainey PB, Travisano M. Adaptive radiation in a heterogeneous environment. Nature. 1998;394:69-72.

39 Nadell CD, Foster KR, Xavier JB. Emergence of spatial structure in cell groups and the evolution of cooperation. PLoS Comput Biol. 2010;6:e1000716.

40 Boddy L. Interspecific combative interactions between wooddecaying basidiomycetes. FEMS Microbiol Ecol. 2000;31:185-94.

41 Ettema CH, Wardle DA. Spatial soil ecology. Trends Ecol Evol. 2002;17:177-83.

42 Greig D, Leu JY. Natural history of budding yeast. Curr Biol. 2009;19:R886-90.

43 Excoffier L, Ray N. Surfing during population expansions promotes genetic revolutions and structuration. Trends Ecol Evol. 2008;23:347-51.

44 Travis JM, Münkemüller T, Burton OJ, Best A, Dytham C, Johst K. Deleterious mutations can surf to high densities on the wave front of an expanding population. Mol Biol Evol. 2007;24:2334-43. 